\title{
Yb:YAG single crystal fiber power amplifier for femtosecond sources
}

\author{
Xavier Délen, ${ }^{1, *}$ Yoann Zaouter, ${ }^{2}$ Igor Martial, ${ }^{3}$ Nicolas Aubry, ${ }^{3}$ Julien Didierjean, ${ }^{3}$ Clemens Hönninger, ${ }^{2}$ \\ Eric Mottay, ${ }^{2}$ François Balembois, ${ }^{1}$ and Patrick Georges ${ }^{1}$ \\ ${ }^{1}$ Laboratoire Charles Fabry, Institut d'Optique, CNRS, Université Paris Sud, 2 Avenue Augustin Fresnel, 91127 Palaiseau Cedex, France \\ ${ }^{2}$ Amplitude Systemes, 6 allée du Doyen Georges Brus, Pessac 33600, France \\ ${ }^{3}$ Fibercryst SAS, La Doua-Bâtiment l'Atrium, Boulevard Latarjet, F-69616 Villeurbanne Cedex, France \\ *Corresponding author: xavier.delen@institutoptique.fr
}

Received November 12, 2012; revised December 6, 2012; accepted December 10, 2012;

posted December 10, 2012 (Doc. ID 179807); published January 7, 2013

\begin{abstract}
We demonstrate a versatile femtosecond power amplifier using a Yb:YAG single crystal fiber operating from $10 \mathrm{kHz}$ to $10 \mathrm{MHz}$. For a total pump power of $75 \mathrm{~W}$, up to $30 \mathrm{~W}$ is generated from the double-pass power amplifier. At a repetition rate of $10 \mathrm{kHz}$, an output energy of $1 \mathrm{~mJ}$ is obtained after recompression. In this configuration, the pulse duration is $380 \mathrm{fs}$, corresponding to a peak power of $2.2 \mathrm{GW}$. The $M^{2}$ beam quality factor is better than 1.1 for investigated parameters. (c) 2013 Optical Society of America

OCIS codes: $320.7090,140.3615$.
\end{abstract}

Ultrafast lasers are now a common tool for scientific and industrial applications. Over the past decade, many technological developments of diode-pumped solid-state laser systems have allowed tremendous improvements of their performance, reliability, and cost. In order to achieve high energy per pulse in the femtosecond regime, master oscillator power amplifier systems are commonly used. Regenerative amplifiers based on bulk or thin disk Yb-doped crystals can amplify ultrashort pulses to several tens of millijoules [1,2]. They can provide high gain and high output energy at a low repetition rate, but they are limited in terms of repetition rate to a few hundreds of kilohertz due to the high-voltage-driven switch speed. Ytterbium-doped optical fibers can also be used to amplify ultrashort pulses. Their high surface-to-volume ratio provides good thermal management and allows attainment of high average powers of several hundreds of watts [3]. However, the signal confinement in smallcross-section cores induces nonlinear effects, such as self-phase modulation and self-focusing, which limit the peak power and the pulse energy. Femtosecond pulses with an energy of $2.2 \mathrm{~mJ}$ were obtained using the well-known chirped pulse amplification technique together with large-core-diameter photonic crystal fibers [4]. Another approach consists in amplifying femtosecond pulses directly in $\mathrm{Yb}$-doped crystals using multipass amplifiers without active elements. Although it requires quite complex systems, the slab geometry has proven to be a very successful approach. Up to $1.1 \mathrm{~kW}$ [5] average power and $20 \mathrm{~mJ}$ [6] energy were obtained with an Yb:YAG Innoslab amplifier. Significant improvement of the emission cross section and the thermal conductivity can be observed at cryogenic temperatures in most $\mathrm{Yb}$ doped crystals. $40 \mathrm{~mJ}$ output energy was obtained using a cryogenic Yb:YAG double-pass amplifier [7]]. However, stronger spectral narrowing at low temperatures induces longer optical pulses of several picoseconds. Finally, the single crystal fiber (SCF) concept lies between fibers and crystals and can contribute to original performance for femtosecond systems.

SCFs are long, thin crystal rods with a diameter lower than $1 \mathrm{~mm}$ and a typical length of a few centimeters.
SCFs are designed for free-space propagation of the laser signal, as in bulk lasers together with a guidance of the pump beam, which depends on the pump brightness. This concept has recently been successfully implemented in Yb:YAG lasers and demonstrated continuous emission of $250 \mathrm{~W}$ from an oscillator based on a $1 \%$ doped Yb:YAG SCF pumped by a $600 \mathrm{~W}$ laser diode [8]. This clearly shows the potential of this approach for high-power extraction. Furthermore, Yb:YAG SCF also demonstrated high single-pass gain with very simple amplifier geometries.

In the context of ultrashort pulse amplification, SCF provides several advantages that clearly benefit the amplification of ultrashort and intense pulses. Among them, the mitigation of nonlinear effects thanks to the short length of interaction together with the large beam diameter is probably the most interesting. Also, the high gain per pass prevents the use of a regenerative amplifier and therefore significantly expands the range of operation at a high repetition rate. Consequently, Yb:YAG SCF has attracted attention as a potential simple, robust, and cost effective amplifier of ultrashort pulses. In a proof-ofprinciple experiment, femtosecond pulses were directly amplified from $400 \mathrm{~mW}$ to $12 \mathrm{~W}$ average power in a double-pass architecture while maintaining the duration of the $30 \mathrm{MHz}$ repetition rate pulses below $400 \mathrm{fs}$ [9].

In this Letter, we demonstrate a femtosecond power amplifier using a diode-pumped Yb:YAG SCF operating from $10 \mathrm{kHz}$ to $10 \mathrm{MHz}$. For a total pump power of $75 \mathrm{~W}$ out of a fiber-coupled high-brightness laser diode, up to $30 \mathrm{~W}$ is generated from the double-pass power amplifier. At a repetition rate of $10 \mathrm{kHz}$, an output energy of $1 \mathrm{~mJ}$ is obtained after recompression.

The experimental setup is shown in Fig. 1. The seed source provides a train of stretched and amplified pulses with a repetition rate ranging from $10 \mathrm{kHz}$ to $10 \mathrm{MHz}$, a maximum energy per pulse of $150 \mu \mathrm{J}$, and a maximum average power of $10 \mathrm{~W}$. The optical spectrum is $4.3 \mathrm{~nm}$ at full width at half-maximum (FWHM) and is centered around $1030 \mathrm{~nm}$ to match the gain bandwidth of the Yb:YAG SCF booster placed downstream after optical isolation. A $500 \mathrm{~mm}$ focal length lens (L1) is used to focus 


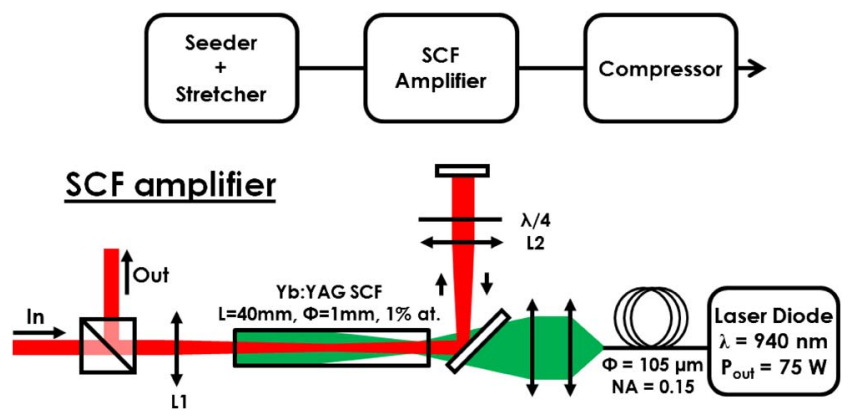

Fig. 1. (Color online) Experimental setup.

the seed beam on the crystal input facet to a measured diameter of $450 \mu \mathrm{m}$. The gain element is a TARANIS laser gain module from Fibercryst, integrating a $40 \mathrm{~mm}$ long, $1 \mathrm{~mm}$ diameter $\mathrm{Yb}$ :YAG SCF with a 1 at. \% doping rate. The two crystal facets have an antireflective coating at the pump and signal wavelengths to prevent parasitic lasing and excess losses at the interfaces. The SCF mount is water-cooled at a temperature of $16^{\circ} \mathrm{C}$. The pump source is a high brightness laser diode (JOLD-75-FC-11 from Jenoptik) with side-by-side combined single emitters. It emits up to $75 \mathrm{~W}$ at $940 \mathrm{~nm}$ coupled in a fiber having a core diameter of $105 \mu \mathrm{m}$ and an NA of 0.15 . The pump fiber output is imaged inside the SCF with a magnification factor of 3.75 using two doublets with focal lengths of 40 and $150 \mathrm{~mm}$. Because of the low NA of the pump beam, it is in free propagation and only weakly guided at the end of the SCF. Depending on the pump waist position, the first section of the SCF in which the pump is in free propagation is between 15 and $25 \mathrm{~mm}$ long. A dichroïc mirror is used to separate the incoming pump beam from the first-pass output of the signal beam. To perform a second pass of amplification into the gain module, the signal waist after the first pass is imaged on a highly reflective plane mirror using a lens (L2) operating in $f-f$ configuration. The adjustment of this imaging system is done at full pump power in order to optimize the second-pass alignment with the nominal thermal lens. The polarization is also rotated by $90^{\circ}$ using a quarterwave plate between the first and second pass, which allows extraction on the input polarizer.

The experiment is first operated at the maximum repetition rate of $10 \mathrm{MHz}$ in order to characterize our amplifier in terms of average power extraction capability in single-pass and double-pass configurations without having to consider nonlinear effects and in particular self-focusing. Figure 2(a) shows the output power versus the pump power for $50 \mathrm{~mW}$ to $5 \mathrm{~W}$ seed power. For a low seed power of $50 \mathrm{~mW}$, gains of 10 and 90 are measured in single-pass and double-pass configurations, respectively. For a $500 \mathrm{~mW}$ input, the output power reaches a maximum of $12 \mathrm{~W}$ in two passes for $72 \mathrm{~W}$ of pump power. In this configuration $95 \%$ of the pump power is absorbed. In a previous experiment, we obtained similar performance, but with a much higher pump power of $180 \mathrm{~W}$ coupled in a $200 \mu \mathrm{m}$ core diameter fiber with an NA of 0.22 [9]. This considerable efficiency improvement is brought by the high pump brightness, which allows for a better geometrical overlap between the signal and the pump beams in the crystal section where the pump is not guided. For a seed power of $5 \mathrm{~W}$, the output power
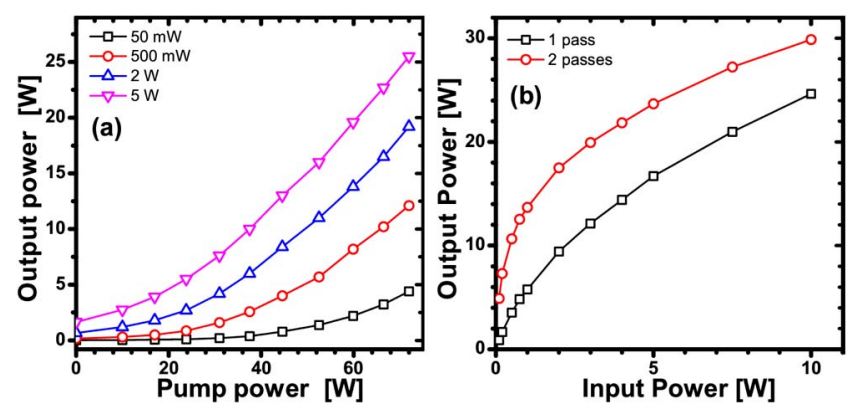

Fig. 2. (Color online) (a) Output power before compression versus pump power for different seed powers in the doublepass configuration and (b) output power versus input power at $10 \mathrm{MHz}$ in single-pass and double-pass configurations for a pump power of $72 \mathrm{~W}$.

reaches 17.8 and $25.5 \mathrm{~W}$ for one pass and two passes, respectively. With an extracted power of $20.5 \mathrm{~W}$, the optical-to-optical efficiency is $28.5 \%$ with the double-pass configuration. Moreover, the slope efficiency increases constantly with the pump power to reach almost $50 \%$. This clearly demonstrates the potential for the use of our amplifier with higher pump powers.

Next, we study the output power of the SCF amplifier as a function of the seed power. It is worth mentioning that without injection, the output signal was not measurable with our power meter for the double-pass configuration (it is estimated to be lower than $2 \mathrm{~mW}$ ). Consequently, the SCF can operate at very low seed power without perturbation by amplified spontaneous emission. Thanks to the high gain available in the SCF amplifier, the output power rapidly reaches the $10 \mathrm{~W}$ level for a seed power of $1 \mathrm{~W}$ [Fig. 2(b)]. As a result, the SCF is able to boost a signal between 10 and $30 \mathrm{~W}$ for any input signal in the range between 1 and $10 \mathrm{~W}$.

To explore the flexibility in the repetition rate and thus in the energy of the Yb:YAG SCF booster, we decrease the frequency of the seed laser from $10 \mathrm{MHz}$ to $10 \mathrm{kHz}$. Figure 3 shows the input energy and the output energy after two passes for different repetition rates. For high repetition rates above $100 \mathrm{kHz}$, the gain is constant and equal to 3 . Between 100 and $10 \mathrm{kHz}$, the output pulse energy continuously increases to $1.3 \mathrm{~mJ}$ at $10 \mathrm{kHz}$, corresponding to a gain of 9 .

Figure 4(a) shows the spectrum of the amplified pulses at maximum output energy $(1.3 \mathrm{~mJ})$. The gain-narrowing effect progressively narrows the optical spectrum, which

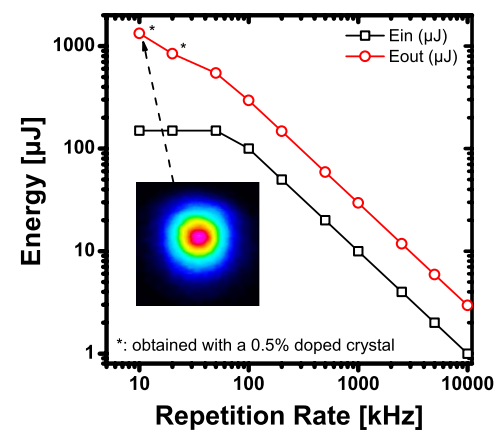

Fig. 3. (Color online) Input and output energies after two passes versus the repetition rate. Inset: output beam profile for $1.3 \mathrm{~mJ}$ at $10 \mathrm{kHz}$ output. 

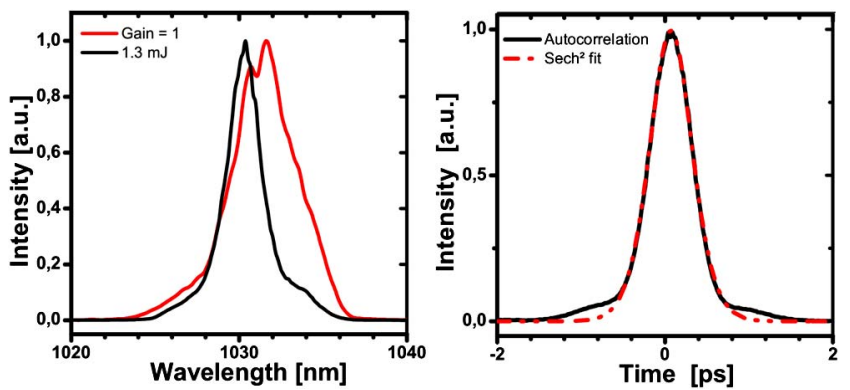

Fig. 4. (Color online) (a) Input and output spectrum, and (b) pulse autocorrelation for $1 \mathrm{~mJ}$ output at $10 \mathrm{kHz}$ after recompression.

FWHM decreases from 4.3 to $2.3 \mathrm{~nm}$ at maximum energy. Consequently, the pulse duration decreases during amplification and is calculated to be about $150 \mathrm{ps}$ at $1.3 \mathrm{~mJ}$, leading to an extracted peak power close to $10 \mathrm{MW}$. This peak power is remarkably high and never could have been reached in a silica-based fiber amplifier, where self-focusing usually destroys the gain medium for a peak power not higher than $4 \mathrm{MW}$. Even if it does not limit the output peak power in our case because of the short crystal length, we can also expect self-focusing in Yb:YAG, inducing a decrease of the beam size on the output facet and potential damage of the antireflective coating. Aware of this effect, we monitor the beam size on the output facet while increasing the seed power at a constant repetition rate. We do not observe any decrease of the beam size that would indicate self-focusing due to higher peak power. Furthermore, no modification of the beam profile is observed, and $M^{2}$ values below 1.1 were measured for all parameters investigated in the article (see beam profile in the inset of Fig. 3).

In order to recover the femtosecond regime, the pulses are recompressed using a transmission-grating-based compressor with an efficiency of $76 \%$. Figure $4(\mathrm{~b})$ shows the autocorrelation trace of the output pulses at a $10 \mathrm{kHz}$ repetition rate and a pulse energy of $1 \mathrm{~mJ}$. It has an FWHM of $590 \mathrm{fs,} \mathrm{corresponding} \mathrm{to} \mathrm{a} \mathrm{pulse} \mathrm{duration} \mathrm{of}$ $380 \mathrm{fs}$, assuming a sech ${ }^{2}$ pulse shape. The corresponding peak power is estimated to be in excess of $2.2 \mathrm{GW}$ after taking into account the fact that more than $95 \%$ of the total energy remains in the central pulse. As expected from the gain narrowing, the pulse duration is increasing for large gain values but it remains below $550 \mathrm{fs}$. Considering the upper state lifetime of $\mathrm{Yb}$ in YAG and the total energy storage capacity, the output energy should continue to increase if we reduce the repetition rate below $10 \mathrm{kHz}$. However, the antireflection coating of the output facet degrades at $1.3 \mathrm{~mJ}$ of extracted energy, corresponding to an energy density of $1.1 \mathrm{~J} / \mathrm{cm}^{2}$ on the output facet. The authors believe that this value can be increased by an order of magnitude by applying different coatings or by using a Brewster-cut gain sample.

In conclusion, we have shown that Yb:YAG SCF pumped by a high-brightness laser diode can be used as a power amplifier for a wide range of seed powers and repetition rates. Sub-550 fs pulses with excellent beam quality $\left(M^{2}<1.1\right)$ have been obtained with average powers between 10 and $23 \mathrm{~W}$ for repetition rates ranging from $10 \mathrm{kHz}$ to $10 \mathrm{MHz}$. The amplifier is also able to produce high energy pulses, the best performance being $1 \mathrm{~mJ}$ pulses at $10 \mathrm{kHz}$ with a duration of $380 \mathrm{fs}$. Considering the versatility of this amplifier and its simplicity and compactness, the Yb:YAG SCF demonstrates a strong potential to boost femtosecond systems in the millijoule range with average powers in the several tens of watts range. Further development of SCF amplifiers will concern power scaling with higher power pump laser diodes and energy scaling with improvement of the damage threshold of the amplifier faces.

$X$. Délen acknowledges the French DGA for the funding of his Ph.D.

\section{References}

1. A. V. Okishev, Opt. Lett. 37, 1199 (2012).

2. T. Metzger, A. Schwarz, C. Y. Teisset, D. Sutter, A. Killi, R. Kienberger, and F. Krausz, Opt. Lett. 34, 2123 (2009).

3. T. Eidam, S. Hanf, E. Seise, T. V. Andersen, T. Gabler, C. Wirth, T. Schreiber, J. Limpert, and A. Tünnermann, Opt. Lett. 35, 94 (2010).

4. T. Eidam, J. Rothhardt, F. Stutzki, F. Jansen, S. Hädrich, H. Carstens, C. Jauregui, J. Limpert, and A. Tünnermann, Opt. Express 19, 255 (2011).

5. P. Russbueldt, T. Mans, J. Weitenberg, H. D. Hoffmann, and R. Poprawe, Opt. Lett. 35, 4169 (2010).

6. M. Schulz, R. Riedel, A. Willner, T. Mans, C. Schnitzler, P. Russbueldt, J. Dolkemeyer, E. Seise, T. Gottschall, S. Hädrich, S. Duesterer, H. Schlarb, J. Feldhaus, J. Limpert, B. Faatz, A. Tünnermann, J. Rossbach, M. Drescher, and F. Tavella, Opt. Lett. 36, 2456 (2011)

7. K. Hong, J. T. Gopinath, D. Rand, A. M. Siddiqui, S. Huang, E. Li, B. J. Eggleton, J. D. Hybl, T. Y. Fan, and F. X. Kärtner, Opt. Lett. 35, 1752 (2010).

8. X. Délen, S. Piehler, J. Didierjean, N. Aubry, A. Voss, M. Abdou Ahmed, T. Graf, F. Balembois, and P. Georges, Opt. Lett. 37, 2898 (2012).

9. Y. Zaouter, I. Martial, N. Aubry, J. Didierjean, C. Hönninger, E. Mottay, F. Druon, P. Georges, and F. Balembois, Opt. Lett. 36, 748 (2011). 